\title{
Micro-tug-of-war: A selective control mechanism for magnetic swimmers
}

\author{
Panayiota Katsamba* and Eric Lauga ${ }^{\dagger}$ \\ Department of Applied Mathematics and Theoretical Physics, \\ University of Cambridge, Cambridge CB3 0WA, United Kingdom.
}

(Dated: June 16, 2016)

\begin{abstract}
One of the aspirations for artificial microswimmers is their application in non-invasive medicine. For any practical use, adequate mechanisms enabling control of multiple artificial swimmers will be of paramount importance. Here we theoretically propose a multi-helical, freely-jointed motor as a novel selective control mechanism. We show that the nonlinear step-out behavior of a magnetized helix driven by a rotating magnetic field can be exploited, when used in conjunction with other helices, to obtain a velocity profile that is non-negligible only within a chosen interval of operating frequencies. Specifically, the force balance between the competing opposite-handed helices is tuned to give no net motion at low frequencies (tug-of-war) while in the middle frequency range the magnitude, and potentially the sign, of the swimming velocity can be adjusted by varying the driving frequency. We illustrate this idea on a two-helix system and demonstrate how to generalize to $N$ helices, both numerically and theoretically. We then explain how to solve the inverse problem and design an artificial swimmer with an arbitrarily-complex velocity vs. frequency relationship. We finish by discussing potential experimental implementation.
\end{abstract}

\section{INTRODUCTION}

From the beginnings of human intellectual activity, scientists and philosophers have been captivated by the beauty hidden in the smallest scales. Technology has now reached the point where micro- and nano- manipulation is not as elusive as it sounded back in Feynman's 1959 lecture "There's plenty of room at the bottom".

Engineering at the micro- or nano-scale includes challenges beyond the mere miniaturising process, primarily from the very physics at these scales [1]. One only needs to zoom down to micrometer resolution and consider something as simple as swimming to appreciate the substantially different physics. In order to self-propel, natural microorganisms need to employ swimming strategies which allow them to go around the constraints set by Purcell's scallop theorem, [2]. Examples include the rotation of a helix [3], whose chiral shape couples rotation to translation, as in most bacteria; the propagation of travelling waves along flexible flagella [4, 5], as in the spermatozoa of many species; or the metachronal wave synchronisation of a carpet of cilia on ciliated organisms [5-7].

Researchers have proposed and constructed a variety of artificial micro- and nano-swimmers often drawing inspiration from natural swimming methods [8]. These are powered either externally [9], often by magnetic fields, or by catalysing a chemical reaction $[10,11]$. The externally powered swimmers proposed so far include helical propellers $[12,13]$ (illustrated in Fig. 1), motors that use flexible filaments [14-16] and surface walkers [17-20].

Following the success of controlling the motion of single artificial swimmers, realistic applications now demand the possibility for multi-device control [8]. Ideally, one

\footnotetext{
* pa335@cam.ac.uk

† e.lauga@damtp.cam.ac.uk
}
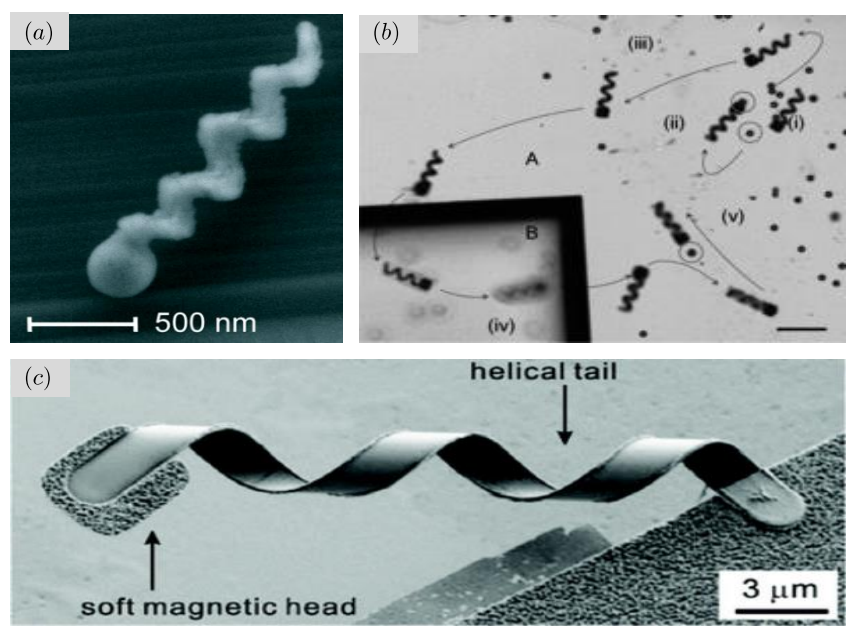

FIG. 1. Experimental realizations of rigid helical swimmers: (a) Chiral silicon dioxide $\left(\mathrm{SiO}_{2}\right)$ colloidal propeller [21]; (b) Pick-and-place micromanipulation of a $6 \mu \mathrm{m}$ diameter microparticle using a helical swimming micromachine, fabricated using 3-D direct laser writing. [22]; (c) SEM of an artificial bacterial flagellum [12] consisting of a ribbon-like helical tail made of a InGaAs/GaAs/Cr trilayer and a soft-magnetic head made of $\mathrm{Cr} / \mathrm{Ni} / \mathrm{Au}$ metal thin films.

would employ the same control inputs to simultaneously move each of these swimmers in a different way, or at least to select and move only a subset of them at a time.

Many research groups have manufactured artificial microswimmers that may be selectively controlled. The stress-engineered MEMS microrobot uses an untethered scratch drive actuator (USDA) and a cantilevered steering arm to move on a elecrode-embedded surface [23]. Selective control of multiple robots is achieved by having different snap-down and release voltage pairs for each robot. The Magmite, designed as a system with intrinsic resonance, uses pulsed magnetic fields to oper- 
ate a magneto-mechanical spring-mass system on a specialised surface [24]. Selective control is achieved by manufacturing each robot to have a different resonant frequency at which it can be operated. In the Mag- $\mu$ Bot, pulsed external magnetic fields induce a stick-slip motion which results in translation. Electrostatic anchoring on a specialised, controlled surface, allows selective control among identical robots by preventing motion [25]. Alternatively, selective control can be achieved with an ordinary, non-specialised surface, by varying the geometrical and magnetic properties of the robots. This exploits the fact that for the stick-slip motion, in which the rectangular-shaped robot needs to be lifted on its edge, to be possible, the magnetic torque must exceed the gravitational rest torque [26].

Propellers driven by external oscillating or rotating magnetic fields offer possibilities for simpler selective control strategies that do not require the presence of a nearby surface. For the achiral three-bead magnetic chain of Ref. [27], altering the field's rotation frequency or strength changes the rotation axis of the microswimmer, giving rise to different modes of motion. Locomotion is only effective for a given range of frequencies of the rotating magnetic field, thus allowing selective control over geometrically similar microswimmers, but with different magnetic properties. Alternatively, one can use the direction of the magnetic moment relative to the long axis of a helical swimmer as a distinguishing control parameter [28]. A multi-step algorithm was proposed that allows independent positioning in which oscillating and rotating fields move a selected motor to a certain location, while the rest still move, but by the time the selected motor has reached its target, they have returned to their initial positions [28]. Finally, recent work used nanohelices with soft magnetic bar and cross-shaped heads [29]. The extra magnetisation axis in the cross-shaped case allows selective control when two types of external magnetic field rotations are used suitably.

The nonlinear step-out behavior of a magnetised chiral structure such as a helix, or screw, which is driven and guided by an externally applied rotating magnetic field has been studied and proposed as a means of providing some selective control [30-35]. When operated below its critical - so called step-out - frequency, the helix rotates synchronously, phase-locked to the rotation rate of the magnetic field, giving rise to a velocity profile which is linear in the operating frequency. At driving frequencies that exceed the step-out frequency, the speed of the helix decays like the inverse power of the driving frequency asymptotically. For a collection of such motors with different step-out frequencies, one can in theory switch between modes where all subgroups are operated (for driving frequencies lower than the minimum step-out frequency), to modes with less and less being operated, by adjusting the driving frequency appropriately. However, from a practical standpoint, it would be important to be able to selectively control any of the different groups, operated if not simultaneously at least one at a time, so that they can be allocated to different tasks.

One of the possible applications of artificial microswimmers, for which selective control is of paramount importance, is that of non-invasive medicine $[8,36]$, one of the greatest aspirations for nano-science. Whether they are to access targeted locations in the body to deliver drugs [37], in which case large numbers of them will be required, or to perform various delicate surgical tasks [38], designing artificial swimmers with adequate multi-robot control is of paramount importance. Other important features that the design should encompass are simplicity and robustness so as to cope in complex operating environments and the possibility of speed adjustment by tuning the control parameters. Given the future need for manufacturing large numbers of these motors, it is also important that the fundamental theoretical analysis is undertaken so that the relationship between the design-parameter space and the resulting microswimmer specification be fully understood. These required design features are not all possible for the current strategies.

In this paper, a multi-helical, freely-jointed motor is proposed and theoretically characterized as a novel selective control mechanism that encompasses all these desired features. The proposed design arose naturally by considering the main problem of interest. In order to selectively control two motors that use the same method of swimming and are powered by the same external signal, they need to respond differently to it, either because they have some different properties (control via variation in the receiver property), or because they are manufactured to only 'listen' to particular sub-signals, e.g. frequencies, that the external signal might consist of (control via distributed signal). Seeking control via variation in the receiver property is ultimately a quest of systems/methods with an intrinsic nonlinearity in the response of the propellers relative to the control parameter of the external signal. The design needs to have enough degrees of freedom so that the nonlinearity manifests a velocity profile that is non-negligible only within an interval of the control parameter. The control parameter could be the frequency of the driving field for example. Then for a collection $\left\{S_{1}, . ., S_{N}\right\}$ of $\mathrm{N}$ sets of such motors, with well-separated effective bands of operational frequencies $\mathcal{B}_{n}=\left(\Omega_{1}^{(n)}, \Omega_{2}^{(n)}\right)$, if the operating frequency $\omega_{h} \in \mathcal{B}_{n}$, the set $S_{n}$ of robots will be controlled, with the rest being stationary or moving at negligible speed.

The nonlinear profile of the single magnetic helix, being close to the desired one except for the lack of a cut-off for low driving frequencies, has inspired us to add more degrees of freedom and consider a motor that consists of two helices of opposite chirality connected in series, which we call a transchiral (i.e. of different chirality) helical motor. The desired cut-off at low frequencies is established by tuning the force balance between the two oppositehanded helices. In isolation, at low frequencies, the two helices would rotate synchronously with the rotation of the magnetic field, but translate in the opposite direction due to the difference in chirality. Assuming that 
they are connected by a joint that allows them to freely rotate relative to one another, the helices will pull each other in opposite directions, a competition resembling tug-of-war. The geometric and magnetic characteristics of the two helices can be chosen such that the net motion of the transchiral helical motor is cancelled in the low frequency regime. For the range of frequencies between the two step-out frequencies, the helix with the highest step-out frequency dominates, giving a net velocity profile that monotonically increases from zero to a maximum value, thereby allowing speed adjustment by varying the driving frequency. Finally, above the maximum step-out frequency, there is negligible locomotion.

Adding more degrees of freedom by considering a multi-helical motor gives rise to more complex banded velocity profiles with extra features, such as bands of negative velocity that enable reversal of the direction of motion by varying the driving frequency. We then derive an approximate analytical model of the full theoretical system, which allows us to solve the inverse problem of prescribing a banded velocity profile and find the geometrical design features that give rise to it.

The paper is organised as follows. After reviewing the physics for the step-out velocity profile of a single magnetised helix, we study the mechanics of multi-body and multi-helical motors. We derive the different velocity profiles attainable by a transchiral and a triple helical motor. An approximate analytical model is then presented, which allows us to solve the inverse problem of prescribing a banded velocity profile and find the geometrical design features that give rise to it. We finish by addressing the issue of experimental implementation and sensitivity of our design to experimental errors.

\section{DESIGN AND LOCOMOTION OF FIELD-DRIVEN HELICAL SWIMMERS}

Magnetised helices guided by a rotating magnetic field can propel effectively in low Reynolds number regimes using two physical ingredients. First, just like a compass needle, a permanent magnet subject to an external magnetic field experiences a torque which tends to align its magnetic moment with the external field. If the latter is constantly rotating, it will continuously apply a torque on the object. The second ingredient is due to the lowReynolds number fluid dynamics around the helix. Because a helix is chiral, when an external torque is applied to it it will not only rotate but also translate along its axis, at a speed which may be found by computing the full resistance matrix of the helical shape, and depends both on its shape and size.

For driving frequencies lower than the step-out frequency, the rotation rate of the helix is phase-locked to that of the field. Above the step-out frequency however, the helix cannot keep up with the field. The phase difference between the two increases, but non-uniformly. During part of the cycle the helix slowly increases its

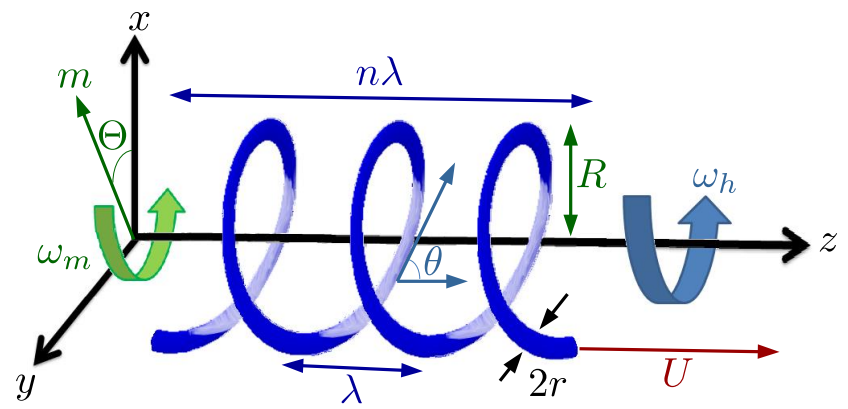

FIG. 2. Geometry of a right-handed helix of wavelength $\lambda$, helix angle $\theta$, radius $R$, diameter of cross-section $2 r$, and number of wavelengths $n$. The magnetic field rotates about the $z$-axis with rotation rate $\omega_{h}$. The helix rotates about the $z$ axis with rotation rate $\omega_{m}$ and translates in the $z$-direction with velocity $U$.

rotation speed, trying to catch up with the field, then effectively dynamically gives up, slows down and starts again. The dynamics of a single magnetised helix giving rise to this nonlinear profile is well understood [30$32,34,35,39]$. Since the single magnetised helix is the fundamental building block of our proposed device and it is its nonlinear behaviour which we wish to exploit for multi-robot control, we shall first review its dynamics in detail.

\section{II.1. Single Helix}

The notation for the helix is shown in Fig. 2. In the frame of a helix, the geometry of its centerline is parametrised by its arc length $\mathbf{x}$ given by

$$
\mathbf{x}=[R \cos (k s \cos \theta), h R \sin (k s \cos \theta), s \cos \theta] .
$$

The long-axis of the helix is assumed to be aligned with the $z$-axis. The main geometrical characteristics are the following: the helicity index $h$ which takes the value +1 or -1 according to whether the helix is right- or lefthanded respectively; the angle $\theta$ between the local tangent and the helix axis (which is constant); the radius $R$ of the helical body (i.e. the radius of the cylinder on which the helix is drawn); the radius $r$ of the cross-section of the wire (we assume it is a circular cross-section); and the number of turns of the helix, $n$. The wavenumber $k$ of the helix is given by $k=2 \pi / \lambda$, where $\lambda$ is the wavelength along the helix axis. The values of $R, k$ and $\theta$ are related via the relationship $\cos ^{2} \theta=\left(1+R^{2} k^{2}\right)^{-1}$. The arc-length along a single turn of the helix is given by $\Lambda=\lambda / \cos \theta$, hence a helix with $n$ turns will have a total length $\Delta z=n \lambda$ along the $z$ axis and total arc length $\Delta s=n \Lambda=2 \pi n R / \sin \theta$. 
The helix is taken to be a permanent magnet, with constant magnetic dipole moment $\mathbf{m}$ which is fixed with respect to the helix geometry and perpendicular to its long axis. We write $|\mathbf{m}|=M V$, where $M$ is the remanent magnetization of the helix and $V=\pi r^{2} \Delta s$ is the volume of the magnetised wire. When placed in an external magnetic field, denoted $\mathbf{h}$, it will experience a magnetic torque $\mathbf{T}_{m}=\mu_{0} \mathbf{m} \wedge \mathbf{h}$. If the external magnetic field is rotating about the $z$-axis with angular frequency $\omega_{h} \mathbf{e}_{z}$ and assuming that the helix is long enough to not wobble [39] but instead to remain aligned with the $z$-axis, the applied magnetic torque on the helix will also point along the $z$-axis and the helix will rotate in the $x-y$ plane about the $z$-axis with angular frequency $\omega_{m}$. If we use $\Theta$ to denote the angle between the $x$-axis and $\mathbf{m}$, which rotates with the body, then we have $\omega_{m}=\mathrm{d} \Theta / \mathrm{dt}$, and the angle between $\mathbf{m}$ and $\mathbf{h}$ is equal to $\omega_{h} t-\Theta$, so that we have the torque given by

$$
\mathbf{T}_{m}(t)=\mu_{0}|\mathbf{m}||\mathbf{h}| \sin \left(\omega_{h} t-\Theta\right) \mathbf{e}_{z}
$$

Due to the hydrodynamic rotation-translation coupling property of the helix, it will also translate with velocity $U$ along the $z$-axis.

Resistive-force theory $[40,41]$ may be used to determine the approximate hydrodynamic forces and torques exerted on the helix. In that framework, the force per unit length, $\delta \mathbf{f}_{h y d r}$, exerted by the helix on the fluid, is given by

$$
\delta \mathbf{f}_{h y d r}=c_{\perp} \mathbf{u}-\left(c_{\perp}-c_{\|}\right)(\mathbf{t} . \mathbf{u}) \mathbf{t},
$$

where $\mathbf{u}=U \mathbf{e}_{z}+\omega_{m} \mathbf{e}_{z} \wedge \mathbf{x}$ is the local velocity and $c_{\perp}$ and $c_{\|}$are the resistance coefficients for motion in the directions perpendicular and parallel to the local tangent $\mathbf{t}$ of the centerline. Their ratio $\rho=c_{\|} / c_{\perp} \approx 1 / 2$, not being unity manifests drag anisotropy, which is crucial for propulsion in the zero Reynolds number regime. One can obtain the force per unit length exerted by the helix on the fluid along the $z$-axis, $\delta \mathbf{f}_{h y d r} . \mathbf{e}_{z}$, and the torque per unit length $\delta \mathbf{T}_{h y d r} \cdot \mathbf{e}_{z}=(\mathbf{x} \wedge \delta \mathbf{f}) \cdot \mathbf{e}_{z}$ exerted by the helix on the fluid along the z-axis as

$$
\begin{aligned}
\delta \mathbf{f}_{h y d r} \cdot \mathbf{e}_{z}= & U\left(c_{\|} \cos ^{2} \theta+c_{\perp} \sin ^{2} \theta\right) \\
& -h\left(c_{\perp}-c_{\|}\right) R \omega_{m} \sin \theta \cos \theta \\
\delta \mathbf{T}_{h y d r} \cdot \mathbf{e}_{z}= & R^{2} \omega_{m}\left(c_{\|} \sin ^{2} \theta+c_{\perp} \cos ^{2} \theta\right) \\
& -h\left(c_{\perp}-c_{\|}\right) R U \sin \theta \cos \theta .
\end{aligned}
$$

Since these expressions are uniform along the helix, the total force and torque exerted by the helix on the fluid along the $z$-axis are thus obtained by multiplying the above by the total arc length, i.e. $n \Lambda=2 \pi n R / \sin \theta$.

In the absence of gradients in the external magnetic field, there are no external forces acting on the helix and thus the total hydrodynamic force on the swimmer must be zero, thereby linearly relating $U$ to $\omega_{m}$. The magnetic torque must balance the hydrodynamic torque exerted by the fluid on the helix due to its motion, leading to the governing equation for the rotation rate.
In its non-dimensionalised form, the governing equation for the phase difference between the external field and the helix $\Delta \Theta=\omega_{h} t-\Theta$ is

$$
\frac{\mathrm{d} \Delta \Theta}{\mathrm{d} \tau}=\frac{\omega_{h}}{\Omega_{\text {so }}}-\sin (\Delta \Theta),
$$

where $\Omega_{s o}$ is the step-out frequency given by

$$
\Omega_{s o}=\frac{\mu_{0}|\mathbf{m} \| \mathbf{h}|}{c_{\perp} R^{3}} \frac{\sin \theta\left(\rho \cos ^{2} \theta+\sin ^{2} \theta\right)}{2 \pi n \rho},
$$

and $\tau$ is the non-dimensionalised time, $\tau=\Omega_{\text {so }}$.

Equation (6) is the well-known Adler's equation which, in its more general form, governs the synchronisation behaviour in a multitude of systems across the spectrum of natural sciences. A simple example in mechanics is the overdamped pendulum driven by a constant torque [42]. More sophisticated systems include the synchronisation of the flagella of microorganisms such as Chlamydomonas [43], heart pacemaker cells, oscillating neurons, fireflies flashing in unison and applauding crowds [42, 44]. In our case, Eq. (6) captures the synchronisation dynamics between the magnetised helix and the driving magnetic field. The phase difference between the two evolves dynamically as a non-uniform oscillator.

The non-dimensional time $\Delta \tau$ for the phase difference $\Delta \Theta$ to change by $2 \pi$ is given by

$$
\Delta \tau=\int_{0}^{2 \pi}\left(\frac{\mathrm{d} \Delta \Theta}{\mathrm{d} \tau}\right)^{-1} \mathrm{~d} \Delta \Theta
$$

Writing the average angular frequency as $\left\langle\omega_{m}\right\rangle=2 \pi / \Delta \tau$, one obtains

$$
\left\langle\omega_{m}\right\rangle= \begin{cases}\omega_{h} & \text { if } \omega_{h} \leq \Omega_{s o} \\ \omega_{h}\left[1-\sqrt{1-\left(\Omega_{s o} / \omega_{h}\right)^{2}}\right] & \text { if } \omega_{h}>\Omega_{s o}\end{cases}
$$

The mean velocity profile, being a scalar multiple of $\left\langle\omega_{m}\right\rangle$, follows the same trend, as shown in the top dashed line (black) in Fig. 3 (top curve): It starts off linear and then decays algebraically above the step-out frequency.

\section{II.2. Multi-body motor}

Having reviewed the dynamics of a single helix, we now turn to the coupled motion of multiple bodies and show how to exploit and modify this nonlinear step-out profile to the desired banded profile in a more general setting. Consider a motor that consists of $N$ magnetized components that are connected in series along their long axis by joints, so that neighbouring magnetised components interact with each other by exerting equal and opposite interaction forces and torques to each other, according to Newton's third law of motion. We assume neighbouring magnetised components are at large separations to neglect hydrodynamic interactions (see $§ I I .5$ for discussion), and that the joint connecting them is negligible 
in size, not magnetized, and allows free relative rotation about the long axis.

Each component is taken to be a permanent magnet, with magnetic dipole moment $\mathbf{m}_{i}$ of magnitude $M_{i} V_{i}$, where $M_{i}$ is the remanent magnetization and $V_{i}$ is the volume of the magnetised material (no summation convention is used here). The vector $\mathbf{m}_{i}$ is taken to be fixed and perpendicular to the long axis of the motor.

When placed in an external magnetic field $\mathbf{h}$ rotating with angular velocity $\omega_{h} \mathbf{e}_{z}$, each component of the motor will experience a magnetic torque $\mathbf{T}_{\text {magn }}^{i}=\mu_{0} \mathbf{m}_{i} \wedge \mathbf{h}$, and will rotate about the $z$-axis with angular frequency $\omega_{m_{i}}$. As before we thus have

$$
T_{\text {magn }}^{(i)}=\mu_{0}\left|\mathbf{m}_{i}\right||\mathbf{h}| \sin \left(\omega_{h} t-\Theta_{i}\right) \mathbf{e}_{z},
$$

where $\Theta_{i}$ is the angle between $\mathbf{m}_{i}$ and the $x$-axis, and $\omega_{h} t-\Theta_{i}$ is the angle between $\mathbf{m}_{i}$ and $\mathbf{h}$.

In practice, the components we are thinking of, and will consider below, are helices, but there is no reason not to generalise to a general chiral geometry when formulating the kinematics of our multi-body motor. Assuming that our magnetised component also translates with velocity $U$ along the $z$-axis, then by linearity the hydrodynamic forces and torques are related to the velocities and rotation rates as

$$
\left(\begin{array}{c}
\mathbf{F}_{h y d r}^{(i)} \\
\mathbf{T}_{h y d r}^{(i)}
\end{array}\right)=\left(\begin{array}{cc}
\mathbf{A}^{(i)} & \mathbf{B}^{(i)} \\
\mathbf{B}^{(i) T} & \mathbf{D}^{(i)}
\end{array}\right)\left(\begin{array}{c}
\mathbf{U}^{(i)} \\
\boldsymbol{\Omega}^{(i)}
\end{array}\right),
$$

where $\mathbf{F}^{(i)}$ and $\mathbf{M}^{(i)}$ are defined as the force and torque that the $i^{\text {th }}$ component exerts on the fluid when it is translating at velocity $\mathbf{U}^{(i)} \equiv U \mathbf{e}_{z}$, common for all components, and rotating at angular velocity $\boldsymbol{\Omega}^{(i)}$. Along the $z$-axis we thus have the linear relationships

$$
\begin{aligned}
& F_{h y d r}^{(i)}=A^{(i)} U+B^{(i)} \frac{\mathrm{d} \Theta_{i}}{\mathrm{~d} t}, \\
& T_{h y d r}^{(i)}=B^{(i)} U+D^{(i)} \frac{\mathrm{d} \Theta_{i}}{\mathrm{~d} t} .
\end{aligned}
$$

The force of interaction between neighbouring components is not necessarily zero, however in the overall force balance, all interaction forces will cancel out and the total force will be zero. Our assumption about the free rotational joint allows us to consider the torque balance for each of the components separately. The system is thus subject to

$$
\sum_{i=1}^{N} F_{h y d r}^{(i)}=0, \quad T_{h y d r}^{(i)}=T_{m a g n}^{(i)} \quad \forall i .
$$

We proceed by non-dimensionalising the problem. Non-dimensional quantities are denoted by hat and nondimensionalised time by $\tau$. Let $M$ be some typical magnetisation and $V$ a typical volume of magnetised material and write $M^{(i)}=M \hat{M}^{(i)}$ and $V^{(i)}=V \hat{V}^{(i)}$. Using a typical length scale $R$, and time scale $\Omega^{-1}$ given by

$$
\Omega=\frac{\mu_{0}|\mathbf{h}| M}{c_{\perp}} \frac{V}{R^{3}},
$$

we have that $U \sim R \Omega, F_{h y d r} \sim c_{\perp} R^{2} \Omega, T_{h y d r} \sim c_{\perp} R^{3} \Omega$, hence $A \sim c_{\perp} R, B \sim c_{\perp} R^{2}$ and $D \sim c_{\perp} R^{3}$.

The force balance, in its non-dimensionalised form, gives

$$
\hat{U}=-\frac{1}{\sum_{k} \hat{A}^{(k)}} \sum_{j=0}^{N} \hat{B}^{(j)} \frac{\mathrm{d} \Theta_{j}}{\mathrm{~d} \tau},
$$

where the angles $\Theta_{i}$ obey the coupled dynamics

$$
\begin{array}{r}
\hat{D}^{(i)} \frac{\mathrm{d} \Theta_{i}}{\mathrm{~d} \tau}-\frac{\hat{B}^{(i)}}{\sum_{k} \hat{A}^{(k)}} \sum_{j=1}^{N} \hat{B}^{(j)} \frac{\mathrm{d} \Theta_{j}}{\mathrm{~d} \tau} \\
=\hat{M}^{(i)} \hat{V}^{(i)} \sin \left(\frac{\omega_{h}}{\Omega} \tau-\Theta_{i}\right) .
\end{array}
$$

This can be written in matrix form as

$$
\sum_{k=1}^{N} \alpha_{i k} \frac{\mathrm{d} \Theta_{k}}{\mathrm{~d} \tau}=\hat{M}^{(i)} \hat{V}^{(i)} \sin \left(\frac{\omega_{h}}{\Omega} \tau-\Theta_{i}\right),
$$

where the matrix $\boldsymbol{\alpha}$ is defined as:

$$
\alpha_{i k}=\left\{\begin{array}{cc}
\hat{D}^{(i)}-\frac{\hat{B}^{(i) 2}}{\sum_{l} \hat{A}^{(l)}} & \text { if } k=i, \\
-\frac{\hat{B}^{(i)} \hat{B}^{(k)}}{\sum_{l} \hat{A}^{(l)}} & \text { if } k \neq i .
\end{array}\right.
$$

\section{II.3. Multi-helical motor}

Having formulated our model in terms of a general chiral structure, we now focus on multi-helical motors, in which the magnetised components are helices.

\section{II.3.1. Equations}

Let $R$ be a typical helical radius, and use that length scale to non-dimensionalise the problem, and $r$ a typical radius of the cross section of a filament so that the typical wire volume is $V=\pi r^{2} R$. The $i^{\text {th }}$ helix has helicity index $h_{i}$, angle $\theta_{i}$, radius $\hat{R}_{i} R$, wavenumber $n_{i}$ and is made out of a wire of cross-sectional radius $\hat{r}_{i} r$, and of total non-dimensionalised arc length $\Delta \hat{s}_{i}=2 \pi n_{i} \hat{R}_{i} / \sin \theta_{i}$ and magnetised volume $\hat{V}^{(i)}=\hat{r}_{i}^{2} \Delta \hat{s}_{i}$. The helix is assumed to have drag coefficient $c_{\perp}^{(i)}=c_{\perp} \hat{c}_{\perp_{i}}$, where $c_{\perp}$ is a typical resistance coefficient, and $\rho_{i}=c_{\|_{i}} / c_{\perp_{i}} \approx 1 / 2$.

For a helix, we can use equations 4 and 5 to directly quote $\hat{A}^{(i)}, \hat{B}^{(i)}$ and $\hat{D}^{(i)}$ as

$$
\begin{aligned}
& \hat{A}^{(i)}=\Delta \hat{s}_{i} \hat{c}_{\perp_{i}}\left(\rho_{i} \cos ^{2} \theta_{i}+\sin ^{2} \theta_{i}\right) \\
& \hat{B}^{(i)}=-\Delta \hat{s}_{i} \hat{R}_{i} \hat{c}_{\perp_{i}} h_{i}\left(1-\rho_{i}\right) \sin \theta_{i} \cos \theta_{i} \\
& \hat{D}^{(i)}=\Delta \hat{s}_{i} \hat{R}_{i}^{2} \hat{c}_{\perp_{i}}\left(\rho_{i} \sin ^{2} \theta_{i}+\cos ^{2} \theta_{i}\right)
\end{aligned}
$$


Substituting these into Eq. (16), for a multi-helical motor, gives

$$
\begin{aligned}
\hat{U} & =\sum_{j=0}^{N} \mathcal{A}_{j} \frac{\mathrm{d} \Theta_{j}}{\mathrm{~d} \tau}, \\
\mathcal{A}_{j} & =\frac{\hat{c}_{\perp_{j}} \Delta \hat{s}_{j} h_{j}\left(1-\rho_{j}\right) s_{j} c_{j} \hat{R}_{j}}{\sum_{k} \hat{c}_{\perp_{k}} \Delta \hat{s}_{k}\left(\rho_{k} c_{k}^{2}+s_{k}^{2}\right)},
\end{aligned}
$$

and the matrix $\boldsymbol{\alpha}$ in equation Eq. (18) is given by

$$
\frac{\alpha_{i k}}{\Delta \hat{s}_{i}}= \begin{cases}\hat{R}_{i}^{2}\left(\rho_{i} s_{i}^{2}+c_{i}^{2}\right)-h_{i}\left(1-\rho_{i}\right) s_{i} c_{i} \hat{R}_{i} \mathcal{A}_{i}, & \text { if } k=i, \\ -h_{i}\left(1-\rho_{i}\right) s_{i} c_{i} \hat{R}_{i} \mathcal{A}_{k}, & \text { if } k \neq i .\end{cases}
$$

Note that repeated indices in the above equation do not imply Einstein summation, and we use the shorthand notation $s_{i} \equiv \sin \theta_{i}, c_{i} \equiv \cos \theta_{i}$.

\section{II.3.2. Numerical results}

The system of $N$ coupled ordinary differential equations of Eq. (18) can be first solved numerically to obtain the average velocity as a function of the driving frequency. Illustrative results are shown in Fig. 3 in the case of a transchiral motor with two helices, where we pick the parameters $\theta_{1}=\theta_{2}=\pi / 4, m_{1}=6, m_{2}=3$, $\hat{r}_{1}=\hat{r}_{2}=1, \hat{R}_{1}=\hat{R}_{1}=1$, and show the frequency vs. velocity relationship for five different helices characterized by $\left(n_{1}, n_{2}\right)=(1-p / 4, p / 4)$, with $p=\{0,1,2,3,4\}$. Note that in principle the drag coefficients depend on the dimensions of the helices and can thus vary, however the dependence is only logarithmic [40,41], and would require the dimensions of different helices to be orders of magnitude different. We thus assume a constant value of the drag coefficients, which is taken out in the nondimensionalisation process, and take $\perp_{j}=1 \forall j$.

Clearly the addition of one more helix provides extra degrees of freedom for the transchiral motor by altering the standard single helix step-out profile, and the computational results confirm our original intuition to exploit the competition between the two opposite-handed helices. In Fig. 3 we observe the velocity profile transitions from that of a single right-handed helix (top dashed line, black), to that of a single left-handed helix (bottom dashed line, green), via a series of intermediate stages. In all cases, the swimming speeds always starts off proportional to the driving frequency, before reaching each of the step-out frequencies consequently. Varying $\left(n_{1}, n_{2}\right)$ alters the initial slope - most notably, there is a special combination that gives rise to zero slope and hence the banded profile (middle solid line, red). In that case, the transchiral motor has a clear band of operating frequencies outside of which it either does not move (low frequencies) or is very inefficient (high frequencies).

For a triple helical motor, with $\left(h_{1}, h_{2}, h_{3}\right)=$ $(+1,-1,+1)$, as shown in Fig. 4, varying the relative

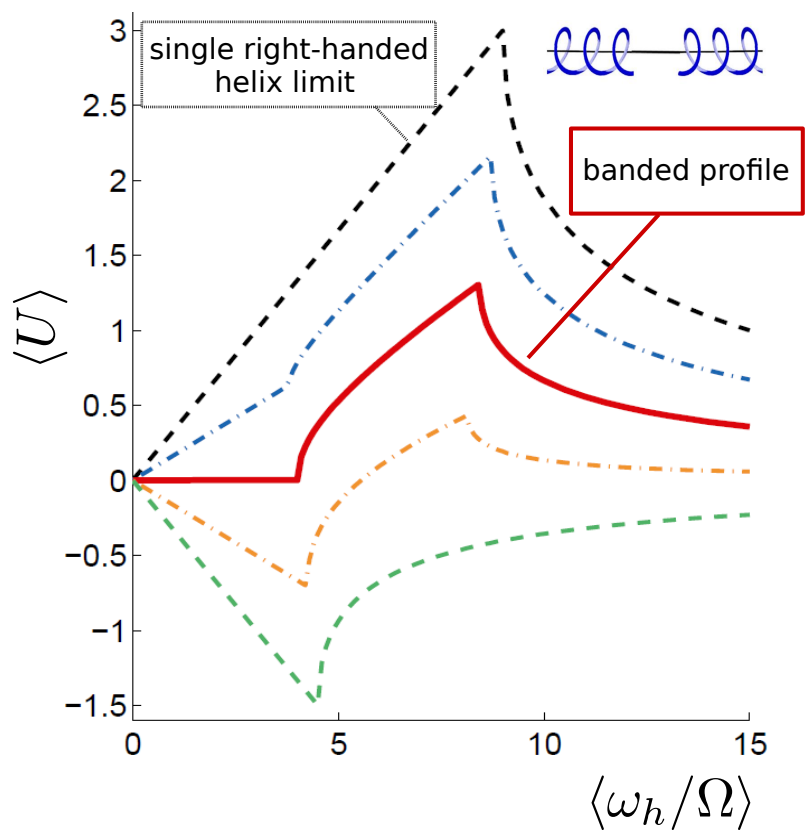

FIG. 3. Mean swimming velocity of two-helix transchiral motor, $\langle U\rangle$, as a function of dimensionless frequency, $\omega_{h} / \Omega$, obtained numerically for the combinations $\left(n_{1}, n_{2}\right)=$ $(1-p / 4, p / 4), p \in 0,1,2,3,4$. All other parameters are kept fixed: $\theta_{1}=\theta_{2}=\pi / 4, \hat{M}_{1}=6, \hat{M}_{2}=3, \hat{r}_{1}=\hat{r}_{2}=1$, and $\hat{R}_{1}=\hat{R}_{1}=1$. We observe the transition from the dynamics of a single right-handed helix (top dashed line, black), to that of a single left-handed helix (bottom dashed line, green), via a series of intermediate stages, including the banded profile (middle solid line, red).

dominances of the helices using different combinations for the number of turns, $n_{1}, n_{2}, n_{3}$, gives rise to various velocity profiles, including a banded velocity profile with frequency ranges with both directionalities (middle solid line, red), thereby allowing reversal of the direction of motion by shifting the driving frequency instead of reversing the direction of rotation of the magnetic field. The general velocity profile for a triple helical motor has three transition points at which each of the helices stepsout, with an initial linear increase in the speed before the first transition point, and a step-out decay after the last one. The limits of a single, right-handed helix $n_{2}, n_{3} \rightarrow 0$, (top dashed line, black) and of a single, left-handed helix $n_{1}, n_{3} \rightarrow 0$, (bottom dashed line, green) are also shown.

\section{II.3.3. Analytical model}

Having characterized our new proposed swimmer numerically, we now show how to use a decoupling approximation to model the dynamics analytically, which we will then exploit to theoretically predict the parameter space for motor design.

Inverting Eq. (18), the system of equations takes the 


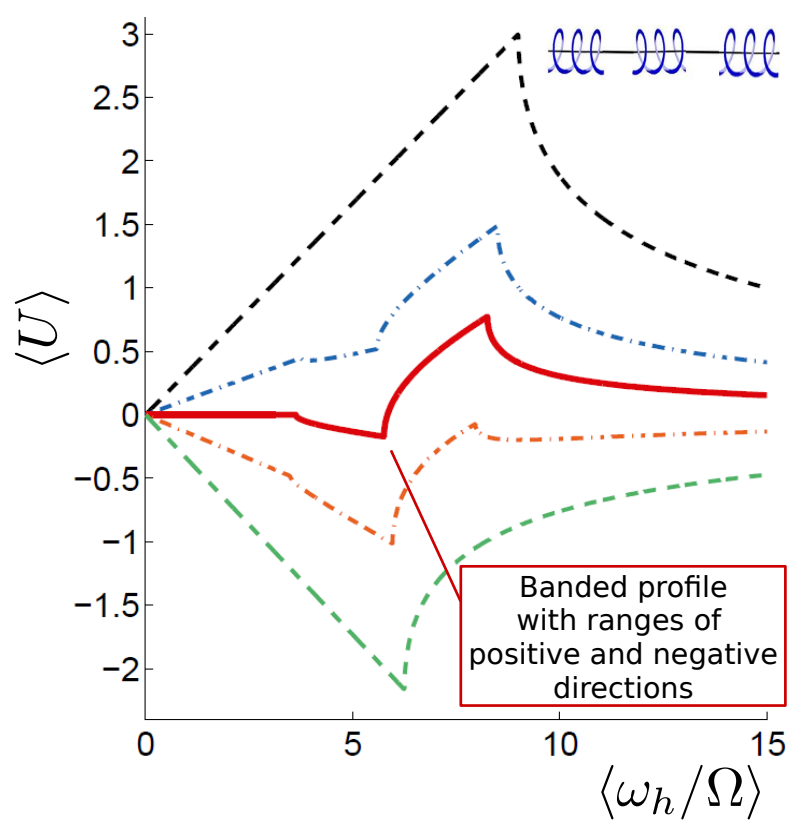

FIG. 4. Propulsion velocity, $\langle U\rangle$, as a function of $\omega_{h} / \Omega$ obtained numerically for a triple helical motor with helicities $\left(h_{1}, h_{2}, h_{3}\right)=(+1,-1,+1)$ for various combinations of the number of turns, whose values, given up to one decimal place are $\left(n_{1}, n_{2}, n_{3}\right)=$ $\{(8,0,0),(6,2.8,1),(4,4.2,1),(2,6,1),(0,8,0)\}$ from top to bottom. All other parameters are kept fixed: $\left(\theta_{1}, \theta_{2}, \theta_{3}\right)=$ $(\pi / 4, \pi / 6, \pi / 5),\left(\hat{M}_{1}, \hat{M}_{2}, \hat{M}_{3}\right)=(6,5,3), \hat{r}_{i}=1$, and $\hat{R}_{i}=1$ for all $i$.

form

$$
\frac{\mathrm{d} \Theta_{i}}{\mathrm{~d} \tau}=f_{i} \sin \Delta \Theta_{i}+\sum_{j \neq i} I_{i j} \sin \Delta \Theta_{j},
$$

with $I_{i j}$ the coupling coefficients (note that $I_{i j}=I_{j i}$ since $\alpha_{i j}$ is symmetric). Noting that the off-diagonal components of $\boldsymbol{\alpha}$ are much smaller than the diagonal ones, we approximate $\alpha_{i j}$ as diagonal and neglect the coupling terms. The system of equations then decouples and we get the approximate system, for all values of $i$

$$
\begin{aligned}
\frac{\mathrm{d} \Delta \Theta_{i}}{\mathrm{~d} \tau} & =\frac{\omega_{h}}{\Omega}-f_{i} \sin \Delta \Theta_{i}, \\
f_{i} & =\frac{\hat{M}^{(i)} \hat{r}_{i}^{2}}{\hat{R}_{i}^{2}\left(\rho_{i} s_{i}^{2}+c_{i}^{2}\right)-h_{i}\left(1-\rho_{i}\right) s_{i} c_{i} \hat{R}_{i} \mathcal{A}_{i}}, \\
\left\langle\frac{\mathrm{d} \Theta_{i}}{\mathrm{~d} \tau}\right\rangle & = \begin{cases}\frac{\omega_{h}}{\Omega} & \text { if } \omega_{h} / \Omega<f_{i}, \\
\frac{\omega_{h}}{\Omega}-\sqrt{\left(\frac{\omega_{h}}{\Omega}\right)^{2}-f_{i}^{2}} & \text { if } \omega_{h} / \Omega>f_{i},\end{cases} \\
& =\frac{\omega_{h}}{\Omega}-\mathbb{1}_{\frac{\omega_{h}}{\Omega}>f_{i}} \sqrt{\left(\frac{\omega_{h}}{\Omega}\right)^{2}-f_{i}^{2}} \\
\langle\hat{U}\rangle & =\sum_{j} \mathcal{A}_{j}\left[f-\mathbb{1}_{f>f_{j}} \sqrt{f^{2}-f_{j}^{2}}\right],
\end{aligned}
$$

where $f=\omega_{h} / \Omega$ is the non-dimensional driving frequency and $\mathbb{1}$ denotes the indicator function $\left(\mathbb{1}_{P}\right.$ equals 1 if the statement $P$ is true and 0 otherwise).

Under these assumptions, the phase-difference between each helix and the magnetic field obeys the non-uniform oscillator equation, Eq. (27), that gives a step-out profile, Eq. (29), with a net velocity which is just the linear superposition of the step-out profiles for each of the rotation rates of the helices, Eq. (31). The quantity $\Omega f_{i}$ is the value of $\omega_{h}$ at which the $i^{\text {th }}$ helix will step-out as part of the multi-helical configuration. Importantly, this is different from the step-out frequency for that helix in isolation since all other helices appear in the sum in the denominator of $\mathcal{A}_{i}$.

Let us now assume that our $N$ helices are numbered in order of increasing values of $f_{i}$. Then the behaviour of the multi-helical motor will be determined by the $N$ transition points $\left(f_{i}, \hat{U}_{i}\right)$ of the $\langle\hat{U}\rangle$ vs. $f$ plot, at which the $i^{\text {th }}$ helix steps-out, where $\hat{U}_{i}$ is given by

$$
\hat{U}_{i}=\left(\sum_{j=1}^{N} \mathcal{A}_{j}\right) f_{i}-\sum_{j<i} \mathcal{A}_{j} \sqrt{f_{i}^{2}-f_{j}^{2}} .
$$

The set $\left\{\left(f_{i}, \hat{U}_{i}\right)\right\}$ then fully determines our design parameter space. Noting that the average non-dimensional velocity increases linearly with the operating frequency until we reach the point

$$
\left(f_{1}, \hat{U}_{1}=f_{1} \sum_{j=1}^{N} \mathcal{A}_{j}\right),
$$

allows us to choose the geometrical parameters of our helices such that

$$
\sum_{j=1}^{N} \mathcal{A}_{j}=0
$$

With this choice the motor stays stationary when operated at frequencies below $\Omega f_{1}$, and is effectively operated within the band $f \in\left(f_{1}, f_{N}\right)$, of width $f_{N}-f_{1}$. Furthermore, its velocity at the transition points is simply given by

$$
\begin{gathered}
\hat{U}_{i}=-\sum_{j<i} \mathcal{A}_{j} \sqrt{f_{i}^{2}-f_{j}^{2}} . \\
\text { II.3.4. Double helical motor }
\end{gathered}
$$

We illustrate the accuracy of our analytical approach with multi-helical motors composed of two and three helices. With two helices, the motor manifests an effective band of frequencies $\left(f_{1}, f_{2}\right)$ of width $f_{2}-f_{1}$. For $f<f_{1}$, the artificial swimmer is constructed to be stationary, and for $f \in\left(f_{1}, f_{2}\right)$ it moves at a speed which increases monotonically with $\omega_{h}$, whereas above $f_{2}$, since both helices have stepped-out, it moves at a negligible velocity that decreases as the inverse power of $\omega_{h}$. 


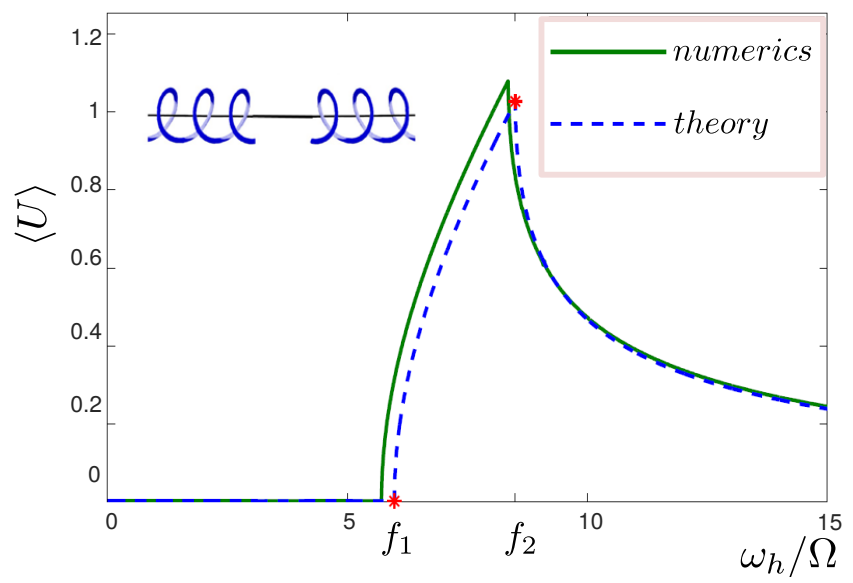

FIG. 5. Mean swimming velocity, $\langle U\rangle$, as a function of $f=$ $\omega_{h} / \Omega$, obtained numerically (solid line, green) and predicted by the theoretical approximation (dashed line, blue), for a double helical motor designed to have zero net motion at low frequencies. The helical parameters are: $\theta_{1}=\pi / 4, \theta_{2}=\pi / 6$, $n_{1}=6.13, n_{2}=5, \hat{M}_{1}=6, \hat{M}_{2}=5, \hat{r}_{1}=\hat{r}_{2}=1, \hat{R}_{1}=\hat{R}_{1}=$ 1 The two critical frequencies are $f_{1} \approx 6$ (with $\hat{U}_{1}=0$ ) and $f_{2} \approx 8.5\left(\hat{U}_{2} \approx 1\right)$.

The comparison between the full numerics and the analytical model is shown in Fig. 5. The blue dashed line shows the profile predicted analytically while the green solid line shows the full computational result without the decoupling approximation. The simple theory is successful at capturing the dynamics of the system, and more importantly, allows us to construct a design parameter space for the motor. Indeed, finding the geometrical parameters that give rise to the banded profile is no longer a 'tuning' process via repeated numerical simulation. Since we have $\hat{c}_{\perp_{j}} \approx 1$ and $\rho_{j} \approx 0.5(j=1,2)$, one just needs to choose the geometrical parameters so as to satisfy $\mathcal{A}_{1}+\mathcal{A}_{2}=0$ which reduces to the simple relationship

$$
n_{1} \hat{R}_{1}^{2} \cos \theta_{1}=n_{2} \hat{R}_{2}^{2} \cos \theta_{2}
$$

Since the denominator in Eq. 28 only depends on $n_{i}$, $R_{i}$, and $\theta_{i}(i=1,2)$, for any combination of these that satisfies this criterion, the critical frequencies $f_{1}, f_{2}$ can be readily set to any value by choosing $\hat{M}_{i}, \hat{r}_{i}$ accordingly.

\section{II.3.5. Triple helical motor}

An additional design feature one might desire is the ability to reverse the direction of motion of the motor by changing the operating frequency alone. This could be achieved with the use of a helical motor composed of three helices. A suitable choice of parameters would allow to split the effective frequency band $\left(f_{1}, f_{3}\right)$, into two bands, $\mathcal{B}_{+}=\left(f_{1}, f_{0}\right)$ and $\mathcal{B}_{-}=\left(f_{0}, f_{3}\right)$, of opposite directionality with $\langle\hat{U}\rangle$ positive in $\mathcal{B}_{+}$and negative in $\mathcal{B}_{-}$, where $f_{0} \in\left(f_{2}, f_{3}\right)$ is such that $\left.\langle\hat{U}\rangle\right|_{f_{0}}=0$ and is

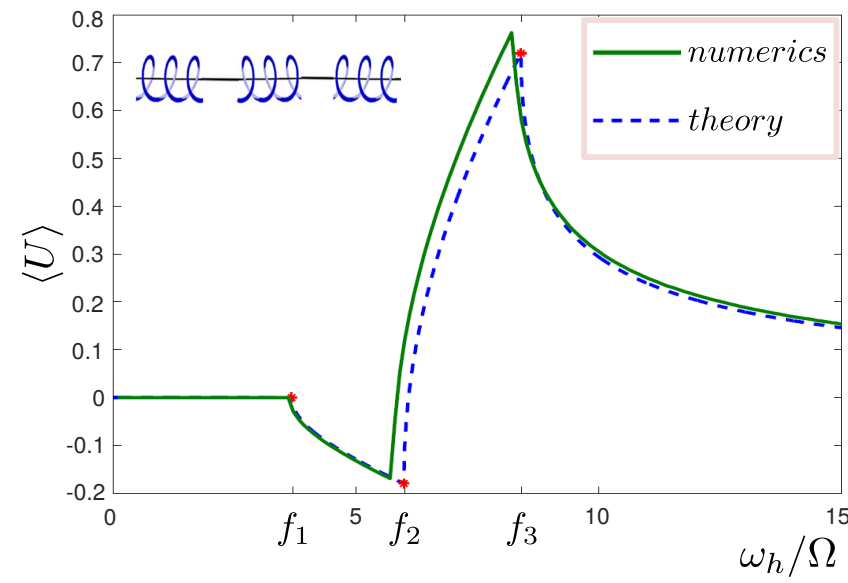

FIG. 6. Mean velocity $\langle U\rangle$ as a function of $f=\omega_{h} / \Omega$, found numerically (solid line, green) and predicted analytically (dashed line, blue), for a triple helical motor. The parameters used are: $\left(h_{1}, h_{2}, h_{3}\right)=(1 ;-1 ; 1),\left(\theta_{1}, \theta_{2}, \theta_{3}\right)=$ $(\pi / 4, \pi / 6, \pi / 5) \quad\left(n_{1}, n_{2}, n_{3}\right)=(4,4.2,1), \quad\left(\hat{M}_{1}, \hat{M}_{2}, \hat{M}_{3}\right)=$ $(6,5,3), \hat{r}_{i}=\hat{R}_{i}=1 \forall i$, The resulting critical points have: $\left(f_{1}, f_{2}, f_{3}\right) \approx(3.7,6,8.4)$ and $\left(\hat{U}_{1}, \hat{U}_{2}, \hat{U}_{3}\right) \approx(0,-0.18,0.72)$.

given by

$$
f_{0}=\sqrt{\frac{\mathcal{A}_{1}^{2} f_{1}^{2}-\mathcal{A}_{2}^{2} f_{2}^{2}}{\mathcal{A}_{1}^{2}-\mathcal{A}_{2}^{2}}} .
$$

With these choices, the motor is stationary for $f \in$ $\left(0, f_{1}\right)$. Then for $f \in\left(f_{1}, f_{2}\right)$ it moves in the negative direction and the speed magnitude increases monotonically from 0 to $\left|\hat{U}_{2}\right|$ as $f$ increases. As $f$ further increases from $f_{2}$ to $f_{3}$, the velocity increases monotonically from its most negative value, $\hat{U}_{2}$, passing through 0 at $f_{0}$, to its most positive value, $\hat{U}_{3}$, at $f_{3}$. For $f$ larger that $f_{3}$, all three helices have stepped out giving rise to negligible velocity that decreases as the inverse of $f$.

The design parameter space of a triple helical motor thus consists of: (a) the boundaries $f_{1}$ and $f_{3}$ of the effective frequency band, (b) the widths $\Delta f_{+}=f_{0}-f_{1}$ and $\Delta f_{-}=f_{3}-f_{0}$ of the positive and negative bands $\mathcal{B}_{+}$and $\mathcal{B}_{-}$respectively, and (3) the most negative and most positive velocities, $\hat{U}_{2}$, and $\hat{U}_{3}$, which occur at $f_{2}$ and $f_{3}$ respectively.

The parameters $\left\{\theta_{i}, n_{i}, \hat{R}_{i}, \hat{r}_{i}, \hat{M}_{i}\right\}(1 \leq i \leq 3)$ can be chosen independently and arbitrarily. An example of a velocity profile obtained by choosing parameters suitable to enable two opposite directionality bands is shown in Fig. 6. Here again, the analytical approach compares very favorably with the full numerics.

\section{II.4. Designing motors with prescribed response functions}

An important question for any engineering system is whether it is possible to solve the inverse problem and 

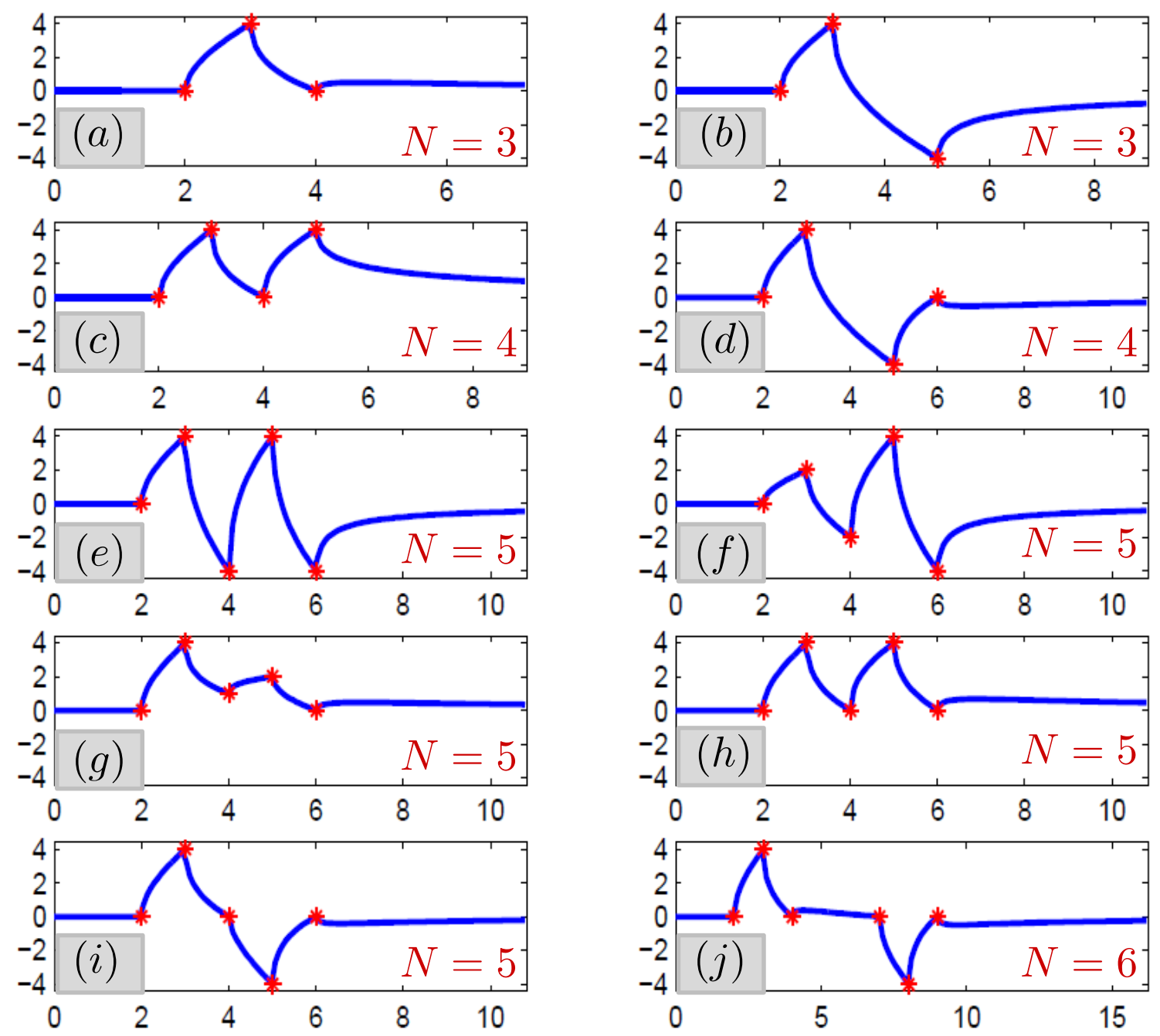

FIG. 7. Various banded design profiles with $N=3,4,5$ or 6 helices. The frequencies $f_{i}$ of the critical transition points, shown in red stars, and the corresponding average propulsion velocities, $\hat{U}_{i}$, are set arbitrarily by the designer. The design features $\left\{\theta_{i}, n_{i}, \hat{R}_{i}, \hat{r}_{i}, \hat{M}_{i}\right\}(1 \leq i \leq N)$ were calculated using the analytical algorithm, and the resulting velocity profiles, shown as blue solid lines, were plotted according to Eq. (23).

find the particular design leading to a pre-determined response function. In our case, this consists of finding the number of helices, $N$, and the values of the parameters $\left\{\theta_{i}, n_{i}, \hat{R}_{i}, \hat{r}_{i}, \hat{M}_{i}\right\}(1 \leq i \leq N)$ which will give rise to a given banded velocity profile, with $\hat{U}_{1}=0$, and with frequencies of the critical transition points, $f_{i}$, and the corresponding average velocities, $\hat{U}_{i}$, set arbitrarily by the designer. We show below that it is possible to construct a simple algorithm to solve this inverse problem.

For simplicity we take the helices to all have the same angle, $\theta_{i}=\theta$, and use the approximation $\hat{c}_{\perp_{j}} \approx 1, \rho_{j}=$ $\rho \approx 0.5(j=1,2)$. In the analytical model, expression 24 takes the simpler form

$$
\mathcal{A}_{j}=\frac{h_{j} \hat{R}_{j}^{2} n_{j}}{\sum_{k} \hat{R}_{j} n_{j}} \frac{(1-\rho) \sin \theta \cos \theta}{\rho \cos ^{2} \theta+\sin ^{2} \theta} .
$$

Noting that the coefficients $\mathcal{A}_{i}$ are independent of $\hat{M}_{i}$, whereas the $f_{i}$ given in Eq. (28) are linear in $\hat{M}_{i}$, means that after all the geometrical features are decided, one can always tune the $f_{i}$ to the desired critical frequencies by choosing the value of $\hat{M}_{i}$ appropriately. Notably, the expressions for the critical velocities $\hat{U}_{i}$ given in Eq. (35) involve only the coefficients $\mathcal{A}_{j}$ with $j<i$ and the values $f_{i}$, and thus using the chosen values for the $f_{i}$ 's, one can solve for the coefficients $\mathcal{A}_{j}$ iteratively: the value of $\hat{U}_{2}$ determines $\mathcal{A}_{1}$, that of $\hat{U}_{3}$ determines $\mathcal{A}_{2}$ etc. until $\hat{U}_{N}$ 
which determines $\mathcal{A}_{N-1}$. The iterative formula is given by

$$
\mathcal{A}_{l-1}=-\frac{\hat{U}_{l}+\sum_{k=1}^{l-2} \mathcal{A}_{k} \sqrt{f_{l}^{2}-f_{k}^{2}}}{\sqrt{f_{l}^{2}-f_{l-1}^{2}}},
$$

for $l=2, \ldots, N$. Then the value of $\mathcal{A}_{N}$ is chosen as

$$
\mathcal{A}_{N}=-\sum_{j=1}^{N-1} \mathcal{A}_{j}
$$

in order to satisfy $\hat{U}_{1}=0$. Once the $\mathcal{A}_{i}$ 's are determined, one proceeds to invert the expression

$$
\mathcal{A}_{j}=\frac{h_{j} \hat{R}_{j}^{2} n_{j}}{\sum_{k} \hat{R}_{j} n_{j}} \frac{(1-\rho) \sin \theta \cos \theta}{\rho \cos ^{2} \theta+\sin ^{2} \theta},
$$

in order to solve for $h_{j}, \hat{R}_{j}$, and $n_{j}$. If we choose $n_{j} \hat{R}_{j}=a$ for all $j$, where $a$ is some constant, then Eq. (41) reduces to

$$
\mathcal{A}_{j}=\frac{h_{j} \hat{R}_{j}}{N} \frac{(1-\rho) \sin \theta \cos \theta}{\rho \cos ^{2} \theta+\sin ^{2} \theta} .
$$

The helicity indices are given by $h_{j}=\operatorname{sign}\left(\mathcal{A}_{j}\right)$ so we obtain

$$
\hat{R}_{j}=N\left|\mathcal{A}_{j}\right| \frac{\rho \cos ^{2} \theta+\sin ^{2} \theta}{(1-\rho) \sin \theta \cos \theta}, \quad n_{j}=a / \hat{R}_{j},
$$

and finally the values $\hat{M}_{i}$ are chosen to tune the critical frequencies $f_{i}$ to the desired values

$$
\hat{M}_{i}=f_{i} \frac{\hat{R}_{i}^{2}\left(\rho_{i} s_{i}^{2}+c_{i}^{2}\right)-h_{i}\left(1-\rho_{i}\right) s_{i} c_{i} \hat{R}_{i} \mathcal{A}_{i}}{\hat{r}_{i}^{2}} .
$$

Implementing this algorithm allows the design of almost any banded velocity profile, as demonstrated in Fig. 7. Prescribing the positions of the critical transition points, shown as red stars, is sufficient to determine the profile shown in solid blue line, which is plotted according to Eq. (23). Most notably, three helices allow for profiles with a banded profile were the last transition point is chosen to have zero velocity, as shown in Fig. 7(a). This allows a better drop-off of the velocity for higher frequencies compared to that offered by the transchiral motor of Fig. 5. Four helices combine this advantage with the possibility of frequency ranges with motion in the opposite direction (Fig. 7d). With five helices, the cross-over frequency between these two ranges can be prescribed (Fig. 7i). Six helices allow for separated positive and negative bands with a prescribed separation (Fig. 7j).

\section{II.5. Experimental considerations}

In an experimental setup, the velocity profile of a fabricated multi-helical motor would vary from the designed theoretical estimates above due to a number of possible effects, including errors during the fabrication process, possible friction from the rotational joint, hydrodynamic interactions between the helical components within it and thermal fluctuations. In this section we address these experimental considerations.

\section{II.5.1. Hydrodynamic interactions}

To discuss some of the implications of hydrodynamic interactions, we now use the setup of a tranchiral motor. Consider two helices that are actuated by a rotating magnetic field and coupled via a joint that allows free relative rotation but restricts them to move at the same translational velocity. Assume the two helices are well-separated. The effect of the joint is that the two helices push or pull each other and hence are not force-free (which would be the case had they not been coupled by the joint). Thus each of the helices is subject to the farfield velocity of the other as a point force, or Stokeslet, to leading order (had they been decoupled it would have been a rotlet, or point torque). The far-field of the first helix at a point with position vector $\mathbf{y}$ relative to the first helix is thus given by the Stokeslet term

$$
\mathbf{u}_{\mathbf{1}}{ }^{f a r}=\frac{1}{8 \pi \mu}\left(\frac{\mathbf{1}}{|\mathbf{y}|}+\frac{\mathbf{y y}}{|\mathbf{y}|^{3}}\right) \cdot \mathbf{F}_{\mathbf{1}}{ }^{h y d r},
$$

where $\mathbf{F}_{\mathbf{1}}{ }^{h y d r}$ is the total hydrodynamic force exerted by helix 1, of total arc length $L_{1}$ translating at speed $U_{1}$, to the surrounding fluid and scales as $F_{1}^{h y d r} \sim \mu U_{1} L_{1}$, where $\mu$ is the dynamic viscosity of the fluid. Here we are assuming that the helix is long enough, $L_{1} \gg R_{1}$, so as not to wobble or equivalently the $\mathrm{x}, \mathrm{y}$ components of $\mathbf{F}_{\mathbf{1}}{ }^{\text {hydr }}$ to be negligible compared to the $\mathrm{z}$ component (the ratio of these scales as $R_{1} / L_{1}$ ). The induced far-field flow of helix 1 on helix 2, assuming these are separated by a distance $d \gg R_{1}, R_{2}, L_{1}, L_{2}$, scales as $\left.u_{1}^{f a r}\right|_{2} \sim U_{1} L_{1} / d$. Comparing the velocity field of helix 2 with no hydrodynamic interactions with the far field velocity acting on it due to helix 1 , since $U_{1}=U_{2}$, we obtain

$$
\frac{\left.u_{1}^{\text {far }}\right|_{2}}{u_{2}^{\text {no hydro }}} \sim \frac{U_{1} L_{1} / d}{U_{2}} \sim \frac{L_{1}}{d} .
$$

Similarly for the effect of helix 2 on helix 1 , with indices 1 and 2 exchanged. Therefore, the effect of hydrodynamic interactions can be neglected for $d \gg L_{1}, L_{2}$.

\section{II.5.2. Thermal fluctuations}

The issue of thermal fluctuations affects all microswimmers, both biological and man-made. For any solid body actuated by means of an external force $\boldsymbol{F}$, and an external torque $\boldsymbol{T}$, and moving as a result with with velocity 


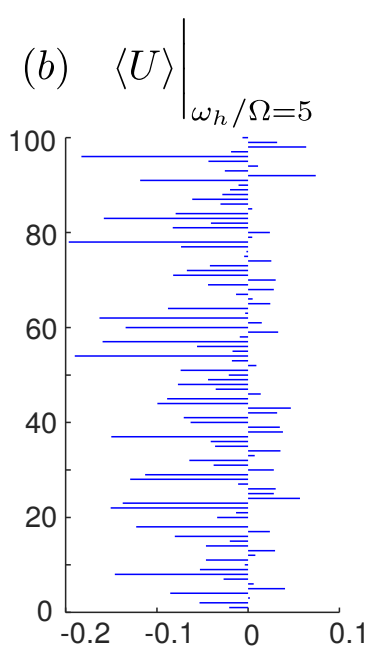

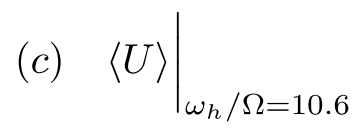

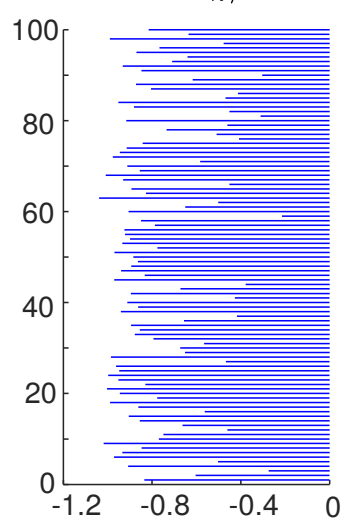

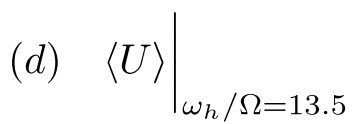

(e) $\left.\langle U\rangle\right|_{\omega_{h} / \Omega=23.5}$
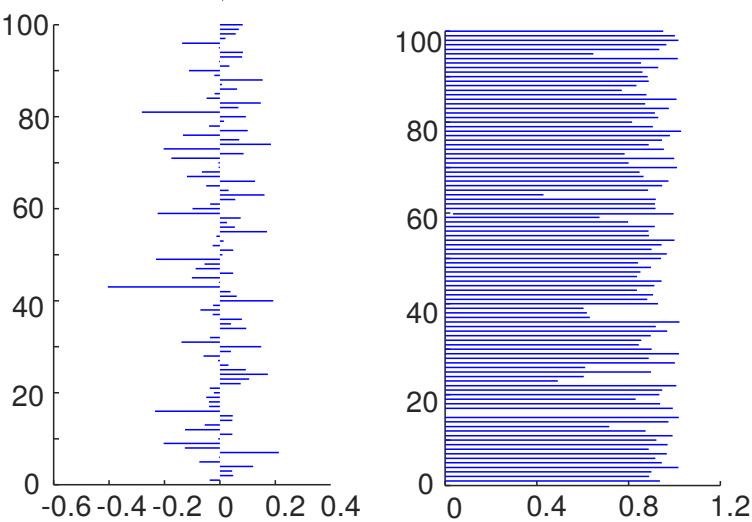
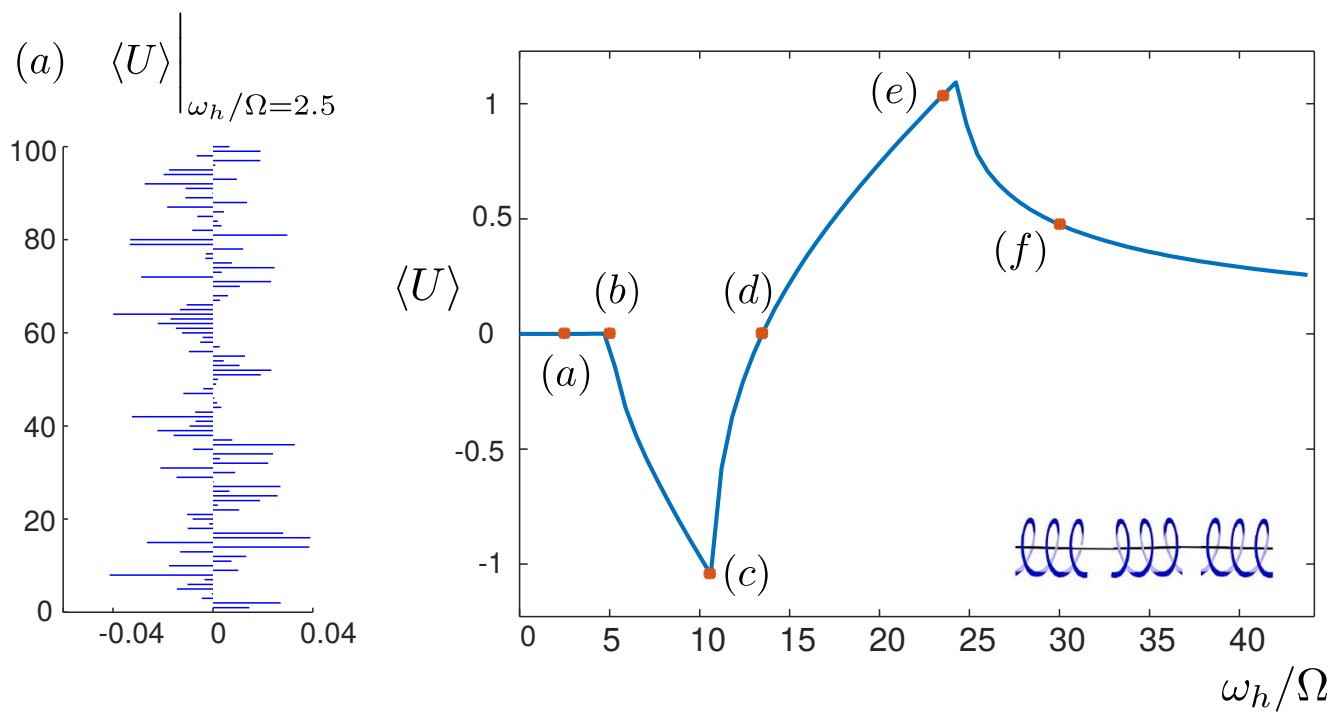
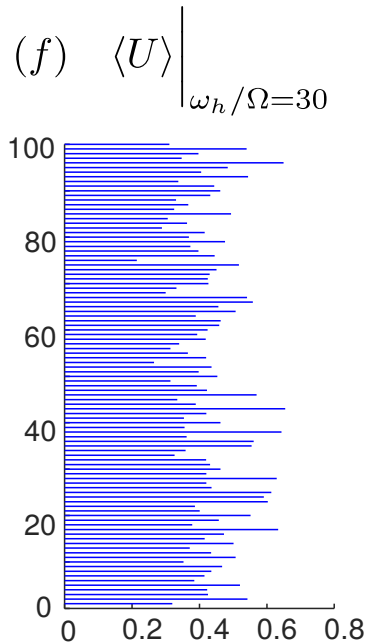

FIG. 8. Simulation of an experiment with experimental errors: The velocities of 100 motors drawn from the same Gaussian distribution with mean $\mu$ equal to the designed value and standard deviation $\sigma$ given by $3 \sigma=0.05 \times \mu$. The designed parameters, were taken to have the following values, shown to one decimal place: $\left(h_{1}, h_{2}, h_{3}\right)=(1 ;-1 ; 1),\left(n_{1}, n_{2}, n_{3}\right)=$ $(4,9.33,6.5),\left(\theta_{1}, \theta_{2}, \theta_{3}\right)=(\pi / 4, \pi / 6, \pi / 5),\left(\hat{M}_{1}, \hat{M}_{2}, \hat{M}_{3}\right)=(18,94.1), \hat{r}_{i}=1, \hat{R}_{i}=1$ for all $i$.

$\boldsymbol{U}$ and rotation rate $\boldsymbol{\Omega}$ given by

$$
\left(\begin{array}{l}
\boldsymbol{U} \\
\boldsymbol{\Omega}
\end{array}\right)=\left(\begin{array}{cc}
\mathcal{M} & \mathcal{N} \\
\mathcal{N}^{T} & \mathcal{O}
\end{array}\right)\left(\begin{array}{l}
\boldsymbol{F} \\
\boldsymbol{T}
\end{array}\right)
$$

the mobility matrix above (which is the inverse of the matrix in Eq. 11) also governs the diffusive behaviour of the body, via the fluctuation-dissipation theorem. Assuming thermal equilibrium at temperature $T$, the translational diffusion constant of a solid body is given by the Stokes-Einstein relationship $\boldsymbol{D}=k_{B} T \mathcal{M}$, where $k_{B}$ is the Boltzmann constant, while the rotational diffusion constant is given by $\boldsymbol{D}_{R}=k_{B} T \mathcal{O}$. For a body with a typical length scale $L$, the constituent submatrices $[\mathcal{M}],[\mathcal{N}],[\mathcal{O}]$ of the mobility matrix scale as $[\mathcal{M}] \sim$ $(\mu L)^{-1}, \quad[\mathcal{N}] \sim\left(\mu L^{2}\right)^{-1},[\mathcal{O}] \sim\left(\mu L^{3}\right)^{-1}[41]$.

Comparing the typical timescales for diffusion induced motion, $\tau_{D} \sim L^{2} /[D]$ and diffusion induced reorientation $\tau_{R} \sim 1 /\left[D_{R}\right],[41]$ with the locomotion induced timescales for translation, $\tau_{\text {trans }} \sim L / U$ and rotation $\tau_{\text {rot }} \sim 1 / \omega$, the two ratios of time scales which have to be small for thermal fluctuations to be neglected are

$$
\frac{\tau_{\text {trans }}}{\tau_{D}} \sim \frac{k_{B} T}{\mu L^{2} U}, \quad \frac{\tau_{r o t}}{\tau_{R}} \sim \frac{k_{B} T}{\mu L^{3} \omega} .
$$

At room temperature, $k_{B} T \sim 10^{-21} \mathrm{~J}$; taking the dynamic viscosity of water $\mu \sim 10^{-3}$ Pas, and a typical frequency of $10 \mathrm{~Hz}$, if we wish these ratios to be of the order of $10^{-2}$, or $10^{-3}$, the motors need to be a few mi- 
crometers in size, which is consistent with the size used in current experimental implementations.

\section{II.5.3. Collection of motors and fabrication errors}

Let us now investigate the effect of fabrication errors, i.e. the fact that the equipment produces motors with errors in their design features, and illustrate how robust the velocity profiles are to such errors.

Assume that one attempted to fabricate a collection of 100 identical multi-helical motors with given geometrical and magnetic features. Such a process would be prone to experimental error, hence as the driving frequency is varied, each of the motor would follow a slightly perturbed velocity profile. Hence if one designs a multi-helical motor to obtain a given velocity profile, and fabricates one such motor with his equipment giving rise to a $5 \%$ error to each of the design features of the motor, the actual velocity profile would deviate from the designed one.

As an indication of this variation, we simulate this numerically by considering 100 realisations of the same design, where the values for the design features are drawn from a Gaussian distribution with mean $\mu$ equal to the designed value and standard deviation $\sigma$ given by $3 \sigma=0.05 \times \mu$. For this, we have used the fact that for the gaussian distribution, the values less than three standard deviations away from the mean account for $99.73 \%$ of the set. The designed parameters, were taken to have the following values, shown to one decimal place: $\left(h_{1}, h_{2}, h_{3}\right)=(1 ;-1 ; 1),\left(n_{1}, n_{2}, n_{3}\right)=(4,9.3,6.5)$, $\left(\theta_{1}, \theta_{2}, \theta_{3}\right)=(\pi / 4, \pi / 6, \pi / 5),\left(\hat{M}_{1}, \hat{M}_{2}, \hat{M}_{3}\right)=(18,94.1)$, $\hat{r}_{i}=1$, and $\hat{R}_{i}=1$ for all $i$.

Ensemble averages of the velocity give us an idea of the deviations from the designed profile a realistic realisation would have. The average velocity of each of these 100 motors for various frequencies is shown in Fig. 8. The velocities of 100 motors drawn from the same Gaussian distribution at a given frequency will have the designed velocity as their mean. Due to variations from the mean, the velocity of an individual propeller can have direction opposite to the expected one, especially for frequencies for which the designed speed is close to zero, as shown in Fig. 8(a,b,d). However, the important theoretical design features are conserved under noisy conditions and with an increase in the frequency the swimmers undergo the transitions in the magnitude of the velocity: small $\rightarrow$ negative $\rightarrow$ small $\rightarrow$ positive $\rightarrow$ small. The idea proposed in this paper should thus be experimentally robust as far as far as fabrication errors are concerned.

\section{II.5.4. Joints}

The basic ingredient of our proposed mechanism is the competition between the two opposite handed helices, and it requires relative rotation between the helices, possibly with friction. A non-zero rotational fric- tion will perturb the velocity profiles (hence we have assumed a friction-free rotational joint for simplicity) but it will not modify the basic physical ideas proposed above. As elusive as they might sound, setups with low rotational friction already exist in Nature, even below the nanometer scale. In molecules, single and triple covalent bonds, e.g. between carbon atoms, do allow free-rotation of the parts of the molecule on either side of the bond, if there are no steric hindrance problems. Perhaps electrostatic interactions between dipole-charged helices could be used to set up an equilibrium distance between them, from which they can rotate relative to each other without contact. Alternatively, one could use a modified version of the 'Christmas' cracker setup of Ref. [45]. In that context, Boron-Nitride nanotubes of different radii with their ends overlapping exhibit ultra-high interlayer friction. Graphene sheets on the other hand, have extremely low friction when sliding past each other [46]. A hybrid design, with nanotubes that have frictional anisotropy in rotation (easy) and translation (difficult), could be a practical solution.

\section{CONCLUSION}

This paper addresses the problem of selective control of multiple artificial swimmers. We began by identifying the need for a design with a suitable intrinsic nonlinearity such that each device can only function within a given band of frequencies. Adding more degrees of freedom by extending the single helix to the multi-helical, freelyjointed motor proposed here, enabled us to exploit the step-out feature to obtain the desired velocity profile.

The velocity profile for a single helix increases linearly with the rotation rate of the magnetic field until it reaches the step-out frequency, after which it decays. In our multi-helical motor, choosing the magnetisation and geometric parameters suitably, net motion can be cancelled for sufficiently low operating frequencies (microtug-of-war) whereas in the high frequency regime where all the helices have stepped-out, motion is negligible. In the middle frequency range, velocity increases monotonically with the driving frequency for the transchiral case. The added-degrees of freedom of the triple-helical motor can be used so that the direction of motion can also be reversed by altering the frequency within the effective band.

A simple approximation enables us to construct a design parameter space to obtain analytical estimates of our design's resulting features. Most notably, these relations are simple enough so that a simple algorithm can be employed to solve the inverse problem: We can choose, prior to experimental fabrication or numerical simulation, the geometric and magnetic parameters of the design that will give rise to the desired banded velocity profile, which we design by prescribing the transition points. With enough helices we have enough degrees of freedom to prescribe a banded velocity profile with 'forwards' and 
'reverse' frequency bands of widths and separation of our choice.

As theorists, we have introduced in this paper the principle of a novel selective control mechanism and discussed experimental constraints and how to limit them. We hope that these ideas will motivate experimental groups to develop practical realisations of the transchiral helical motor.

\section{ACKNOWLEDGEMENTS}

This work was funded in part by the European Union through a Marie Curie CIG grant (E.L.) and by the EPSRC (P.K.).
[1] M. Wautelet, "Scaling laws in the macro-, micro- and nanoworlds," Eur. J. Phys. 22, 601 (2001).

[2] E. M. Purcell, "Life at low reynolds number," Am. J. Phys. 45, 3-11 (1977).

[3] L. Turner, W. S. Ryu, and H. C. Berg, "Real-time imaging of fluorescent flagellar filaments," J. Bacteriol. 182, 2793-2801 (2000).

[4] D. Bray, Cell Movements (Garland, New York, 2000).

[5] C. Brennen and H. Winet, "Fluid mechanics of propulsion by cilia and flagella," Ann. Rev. Fluid Mech. 9, 339398 (1977).

[6] I. R. Gibbons, "Cilia and flagella of eukaryotes." J. Cell Biol. 91, 107s-124s (1981).

[7] M. A. Sleigh, J. R. Blake, and N. Liron, "The propulsion of mucus by cilia," Am. Rev. Resp. Dis. 137, 726741 (1988).

[8] B. J. Nelson, I. K. Kaliakatsos, and J. J. Abbott, "Microrobots for minimally invasive medicine," Ann. Rev. Biomed. Eng. 12, 55-85 (2010).

[9] S. J. Ebbens and J. R. Howse, "In pursuit of propulsion at the nanoscale," "Soft Matter" "6", "726-738" ("2010").

[10] W. F. Paxton, K. C. Kistler, C. C. Olmeda, A. Sen, S. K. St. Angelo, Y. Cao, T. E. Mallouk, P. E. Lammert, and V. H. Crespi, "Catalytic nanomotors: autonomous movement of striped nanorods," J. Am. Chem. Soc. 126, 13424-13431 (2004).

[11] R. Golestanian, T. B. Liverpool, and A. Ajdari, "Designing phoretic micro- and nano-swimmers," New J. Phys. 9, 126 (2007).

[12] L. Zhang, J. J. Abbott, L. Dong, K. E. Peyer, B. E. Kratochvil, H. Zhang, C. Bergeles, and B. J. Nelson, "Characterizing the swimming properties of artificial bacterial flagella," Nano Lett. 9, 3663-3667 (2009).

[13] A. Ghosh and P. Fischer, "Controlled propulsion of artificial magnetic nanostructured propellers," Nano Lett. 9, 2243-2245 (2009).

[14] R. Dreyfus, J. Baudry, M. L. Roper, M. Fermigier, H. A. Stone, and J. Bibette, "Microscopic artificial swimmers," Nature 437, 862-865 (2005).

[15] W. Gao, S. Sattayasamitsathit, K. M Manesh, D. Weihs, and J. Wang, "Magnetically powered flexible metal nanowire motors," J. Am. Chem. Soc. 132, 14403-14405 (2010).

[16] O. S. Pak, W. Gao, J. Wang, and E. Lauga, "Highspeed propulsion of flexible nanowire motors: Theory and experiments," Soft Matter 7, 8169-8181 (2011).

[17] P. Tierno, F. Golestanian, I. Pagonabarraga, and F. Sagués, "Controlled swimming in confined fluids of magnetically actuated colloidal rotors," Phys. Rev. Lett. 101, 218304 (2008).
[18] P. Tierno, O. Güell, F. Sagués, R. Golestanian, and I. Pagonabarraga, "Controlled propulsion in viscous fluids of magnetically actuated colloidal doublets," Phys. Rev. E 81, 011402 (2010).

[19] C. E. Sing, L. Schmid, M. F. Schneider, T. Franke, and A. Alexander-Katz, "Controlled surface-induced flows from the motion of self-assembled colloidal walkers," Proc. Natl. Acad. Sci. U.S.A. 107, 535-540 (2010).

[20] L. Zhang, T. Petit, Y. Lu, B. E. Kratochvil, K. E. Peyer, R. Pei, J. Lou, and B. J. Nelson, "Controlled propulsion and cargo transport of rotating nickel nanowires near a patterned solid surface," ACS Nano 4, 6228-6234 (2010).

[21] A. Ghosh and P. Fischer, "Controlled propulsion of artificial magnetic nanostructured propellers," Nano Lett. 9, 2243-2245 (2009).

[22] S. Tottori, L. Zhang, F. Qiu, K. K. Krawczyk, A. FrancoObregn, and B. J. Nelson, "Magnetic helical micromachines: Fabrication, controlled swimming, and cargo transport," Adv. Mater. 24, 811-816 (2012).

[23] B. R. Donald, C. G. Levey, and I. Paprotny, "Planar microassembly by parallel actuation of mems microrobots," J. Microelectromech. Syst. 17, 789-808 (2008).

[24] D. R. Frutiger, K. Vollmers, B. E. Kratochvil, and B. J. Nelson, "Small, fast, and under control: Wireless resonant magnetic micro-agents," Int. J. Rob. Res. 29, 613636 (2010).

[25] C. Pawashe, S. Floyd, and M. Sitti, "Multiple magnetic microrobot control using electrostatic anchoring," Appl. Phys. Lett. 94, 164108 (2009).

[26] S. Floyd, E. Diller, C. Pawashe, and M. Sitti, "Control methodologies for a heterogeneous group of untethered magnetic micro-robots," Int. J. Rob. Res. 30, 1553-1565 (2011).

[27] U. K. Cheang, F. Meshkati, D. Kim, M. J. Kim, and H. C. Fu, "Multiple-robot drug delivery strategy through coordinated teams of microswimmers," Appl. Phys. Lett. 105, 083705 (2014).

[28] P. Mandal, V. Chopra, and A. Ghosh, "Independent positioning of magnetic nanomotors," ACS Nano 9, 47174725 (2015).

[29] S. Tottori, N. Sugita, R. Kometani, S. Ishihara, and M. Mitsuishi, "Selective control method for multiple magnetic helical microrobots," J. MicroNanomechatronics 6, 89-95 (2011).

[30] E. J. Smith, D. Makarov, S. Sanchez, V. M. Fomin, and O. G. Schmidt, "Magnetic microhelix coil structures," Phys. Rev. Lett. 107, 097204 (2011).

[31] V. M. Fomin, E. J. Smith, D. Makarov, S. Sanchez, and O. G. Schmidt, "Dynamics of radial-magnetized microhelix coils," Phys. Rev. B 84, 174303 (2011). 
[32] V. M. Fomin, E. J. Smith, D. Karnaushenko, D. Makarov, and O. G. Schmidt, "Asymmetric drag in oscillatory motion: Ratchet effect without an asymmetric potential," Phys. Rev. E 87, 052122 (2013).

[33] M. Ishiyama and M. Sendoh K. I. Arai, "Magnetic micromachines for medical applications," J. Magn. Magn. Mater. 41, 242-245 (2002).

[34] B. H. McNaughton, K. A. Kehbein, J. N. Anker, and R. Kopelman, "Sudden breakdown in linear response of a rotationally driven magnetic microparticle and application to physical and chemical microsensing," J. Phys. Chem. B 110, 18958-18964 (2006).

[35] A. W. Mahoney, N. D. Nelson, K. E. Peyer, B. J. Nelson, and J. J. Abbott, "Behavior of rotating magnetic microrobots above the step-out frequency with application to control of multi-microrobot systems." Appl. Phys. Lett. 104, 144101 (2014).

[36] M. Sitti, "Miniature devices: Voyage of the microrobots," Nature 458, 1121-1122 (2009).

[37] W. Gao, D. Kagan, O. S. Pak, C. Clawson, S. Campuzano, E. Chuluun-Erdene, E. Shipton, E. E. Fullerton, L. Zhang, E. Lauga, and J. Wang, "Cargo-towing fuelfree magnetic nanoswimmers for targeted drug delivery," Small 8, 460-467 (2012).
[38] F. Ullrich, C. Bergeles, J. Pokki, O. Ergeneman, S. Erni, G. Chatzipirpiridis, S. Pan, C. Framme, and B. J. Nelson, "Mobility experiments with microrobots for minimally invasive intraocular surgery," Invest. Ophthalmol. Vis. Sci. 54, 2853-2863 (2013).

[39] Y. Man and E. Lauga, "The wobbling-to-swimming transition of rotated helices," Phys. Fluids 25, 071904 (2013).

[40] J. Gray and G. J. Hancock, "The propulsion of sea-urchin spermatozoa," J. Exp. Biol. 32, 802-814 (1955).

[41] E. Lauga and T. R. Powers, "The hydrodynamics of swimming microorganisms," Rep. Prog. Phys. 72, 096601 (2009).

[42] S. H. Strogatz, Nonlinear Dynamics and Chaos (Westview Press, Cambridge, MA, 2000).

[43] K. Y. Wan and R. E. Goldstein K. C. Leptos, "Lag, lock, sync, slip: the many 'phases' of coupled flagella," J. R. Soc. Interface 11, 20131160 (2014).

[44] A. Pikovski, M. Rosenblum, and J. Kurths, Synchronization, A Universal Concept in Nonlinear Sciences (Cambridge University Press, Cambridge, UK, 2001).

[45] A. Nigus, A. Siria, P. Vincent, P. Poncharal, and L. Bocquet, "Ultrahigh interlayer friction in multiwalled boron nitride nanotubes," Nat. Mater. 13, 688-693 (2014).

[46] M. Dienwiebel, G. S. Verhoeven, N. Pradeep, J. W. M. Frenken, J. A. Heimberg, and H. W. Zandbergen, "Superlubricity of graphite," Phys. Rev. Lett. 92, 126101 (2004). 